\title{
Analytical Study of Drift Velocity in Low Dimensional Devices
}

\author{
Mohammad T. Ahmadi ${ }^{1, *}$, Ismail Saad ${ }^{1}$, MunawarA.Riadi ${ }^{1}$, Razali Ismail ${ }^{1}$ and Vijay K.Arora ${ }^{1,2}$ \\ ${ }^{1}$ Faculty of Electrical Engineering University Technology of Malaysia 81310 Johor Skudai \\ ${ }^{2}$ Division of Engineering and Physics, Wilkes University Wilkes-Barre, PA 18766, USA \\ *Author to whom correspondence should be addressed; E-mail: <ahmadiph@gmail.com>
}

Received: 6 November 2008

http://dx.doi.org/10.11113/mjfas.v4n2.49

\section{ABSTRACT}

Understanding of quantum limit in low dimensional devices helps to develop the new device types same as Carbon Nanotube Field Effect Transistor (CNTFET) and Naonowire. For each dimensionality the limitations on carrier drift velocity due to the high-field streaming of otherwise randomly velocity vector in equilibrium is reported. The results are based on the asymmetrical distribution function that converts randomness in zero-field to streamlined one in a very high electric field. The ultimate drift velocity for all dimensions is found to be appropriate thermal velocity for a nondegenerately doped sample of silicon, increasing with the temperature, but independent of carrier concentration. However, the ultimate drift velocity is the Fermi velocity for degenerately doped silicon increasing with carrier concentration but independent of the temperature.

| Drift velocity | Low Dimensional Devices| Quantum Limit |

\section{Introduction}

The quest for high-speed devices and circuits for Ultra-Large-Scale-Integration (ULSI) is continuing. The speed is determined by the ease with which the carrier (electron or holes) can propagate through the length of the device. In the earlier designs, the mobility of the carrier was believed to be of paramount importance. That was the push for Gallium Arsenide (GaAs) considering that the mobility of an electron in GaAs is 5-6 times higher than that of an electron in silicon. However, as development of the devices to nanoscale dimensions continued it became clear that the saturation velocity plays a predominant role. The higher mobility brings an electron closer to saturation as a high electric field is encountered, but saturation velocity remains the same no matter what the mobility. Until today, there is no clear consensus on the interdependence of saturation velocity on low-field mobility that is scattering-limited.

There are a number of theories of high-field transport to answer this interdependence. Among them are Monte Carlo simulations, energy-balance theories, path integral methods, green function and many others. Rigor of mathematics and a number of clandestine parameters that are used in these simulations present a foggy picture of what controls the ultimate saturation of drift velocity. In any solid state device, it is very clear that the band 
structure parameters, doping profiles (degenerate or nondegenerate), and ambient temperatures play a variety of roles in limiting optoelectronic properties. The outcome that higher mobility leads to higher saturation is not supported by experimental observations prompting our careful study of the process controlling the ultimate saturation. In the following, the fundamental processes that limit drift velocity are delineated.

\section{Theory}

The distribution function of the energy $E_{k}$ is given by the Fermi-Dirac distribution function:

$$
f\left(E_{k}\right)=\frac{1}{e^{\frac{E_{k}-E_{F d}}{k_{B} T}}+1}
$$

where $E_{F d}$ is the Fermi energy at which the probability of occupation is half, $d$ is dimension and $T$ is the ambient temperature. In non- degenerately doped semiconductors the ' 1 ' in the denominator of Equation (2.1) is negligible compared to the exponential factor, the distribution is then Maxwellian:

$$
f\left(E_{k}\right)=e^{-\frac{E_{k}-E_{F d}}{k_{B} T}}
$$

This simplified distribution function is extensively used in determining the transport parameters. This simplification is true for non degenerately-doped semiconductors. However, most NANO electronic devices these days are degenerately doped. Hence any design based on the Maxwellian distribution is not strictly correct and often leads to errors in our interpretation of the experimental results. In the other extreme, for strongly degenerate carriers, the probability of occupation is 1 where $E_{k}<E_{F}$ and it is zero if $E_{k}>E_{F}$. Arora [1] modified the equilibrium distribution function of Equation (2.1) by replacing $E_{F d}$ (the chemical potential) with the electrochemical potential $\mathrm{E}_{\mathrm{Fd}}+\mathrm{q} \vec{\varepsilon} \cdot \vec{\ell}$. Here $\vec{\varepsilon}$ is the applied electric field, $\mathrm{q}$ is the electronic charge and $\vec{\ell}$ the mean free path during which carriers are collision free or ballistic. Arora's distribution function is thus given by

$$
f\left(E_{k}\right)=\frac{1}{e^{\frac{E_{k}-E_{F d} \pm q \vec{\varepsilon} \cdot \vec{\ell}}{k_{B} T}}+1}
$$

This distribution has simpler interpretation as given in the tilted band diagram of Figure 1 the carriers at a point $x$ arrive from left or right a mean-free-path $\ell$ away from either side of $x$. It can be seen that the Fermi level on left is $E_{F d}+q \varepsilon \ell$ and that on the right $E_{F d}-q \varepsilon \ell$. These are the two quasi Fermi levels with $E_{F d}$ at the point $x$. The current flow is due to the gradient of Fermi energy $E_{F d}(x)$ when an electric field is applied. 


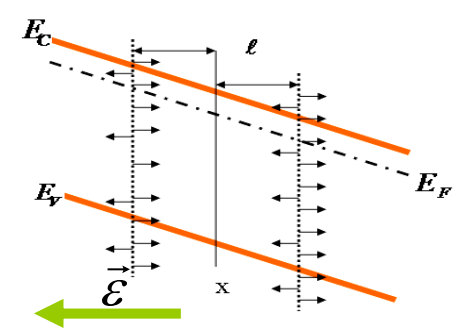

Figure 1: Partial streamlining of random motion of the drifting electrons on a tilted energy band diagram in an electric-field.

Because of this asymmetry in the distribution of electrons, the electrons tend to drift opposite to the electric field $\vec{\varepsilon}$ applied in the negative x-direction (right to left). In an extremely large electric field, virtually all the electrons are travelling in the positive x-direction (opposite to the electric field). This is what is meant by conversion of otherwise completely random motion into a streamlined one. With ultimate velocity per electron equal to $v_{i}$. Hence the ultimate velocity is ballistic independent of scattering interactions.

The ballistic motion in a mean-free path is interrupted by the onset of a quantum emission of energy $\hbar \omega_{0}$. This quantum may be an optical phonon or a photon or any digital energy difference between the quantized energy levels with or without external stimulation present. The mean-free path with the emission of a quantum of energy is related to $\ell_{0}$ (zero-field mean free path) by an expression [2]

$$
\begin{aligned}
& \ell=\ell_{0}\left[1-e^{-\frac{E_{Q}}{q \varepsilon \ell_{0}}}\right]=\ell_{0}\left[1-e^{-\frac{\ell_{Q}}{\ell_{0}}}\right] \\
& q \varepsilon \ell_{Q}=E_{Q}=\left(N_{0}+1\right) \hbar \omega_{0} \\
& N_{0}=\frac{1}{e^{\frac{\hbar \omega_{0}}{k_{B} T}}+1}
\end{aligned}
$$

Here $\left(N_{0}+1\right)$ gives the probability of a quantum emission, and $N_{o}$ is the Bose-Einstein distribution function. The degraded mean free path $\ell$ is now smaller than the low-field mean free path $\ell_{o} \cdot \ell_{\approx} \ell_{o}$ in the ohmic low-field regime as expected. In high electric field, $\ell \approx \ell_{Q}$. The inelastic scattering length during which a quantum is emitted is given by:

$$
\ell_{Q}=\frac{E_{Q}}{q \varepsilon}
$$


Obviously $\ell_{Q}=\infty$ in zero -electric field and will not modify the traditional scattering described by mean free path $\ell_{0}$ as $\ell_{Q}>\ell_{0}$. The low-field mobility and associated drift motion is therefore scattering-limited. The effect of all possible scattering interactions is now buried in the mean free path $\ell_{0}$. However the presence of high electric field makes $\ell_{Q}<<\ell_{0}$. In that extreme we have:

$$
\ell \approx \ell_{Q}=\frac{E_{Q}}{q \varepsilon}
$$

This itself may be enough to explain the degradation of mobility $\mu$ in a high electric field

$$
\mu=\frac{q \tau_{c}}{m^{*}}=\frac{q \ell}{m^{*} v_{i 3}} \approx \frac{q \ell_{Q}}{m^{*} v_{i d}}
$$

Here $\tau_{c}$ is the mean free time in which the electron motion is ballistic. $v_{i d}$ is the mean intrinsic velocity [3] for semiconductors. $v_{i d}$ is the weighted average of $|v|=\sqrt{2 E_{k} / m^{*}}$ with the Fermi-Dirac distribution of Equation (2.1) multiplied by the density of quantum states and is given by

$$
\begin{aligned}
& v_{i d}=v_{t h d} \frac{\mathscr{F}_{(d-1)}^{2}\left(\eta_{d}\right)}{\frac{\mathscr{F}_{(d-2)}}{2}\left(\eta_{d}\right)} \\
& \mathscr{F}_{j}(\eta)=\frac{1}{\Gamma(j+1)} \int_{0}^{\infty} \frac{x^{j}}{e^{(x-\eta)}+1} d x
\end{aligned}
$$

Here, $\mathscr{J}_{j}(\eta)$ is the Fermi-Dirac integral of order $\mathrm{j}$ and $\Gamma(j+1)$ is a Gamma function. The Fermi integral with Maxwellian approximation is always an exponential for all values of $j$ and is given by

$$
\mathscr{F}_{j}(\eta) \approx e^{\eta} \quad \text { (Non-degenerate) }
$$

In the strongly degenerate regime, the Fermi integral transforms to

$$
\mathscr{F}_{j}(\eta) \approx \frac{1}{\Gamma(j+1)} \frac{\eta^{j+1}}{j+1}=\frac{\eta^{j+1}}{\Gamma(j+2)} \quad \text { (Degenerate) }
$$


The ultimate average velocity per electron is now $v_{i d}$ and is a function of temperature and doping concentration $[4,5]$

$$
v_{i d}=v_{t h} \frac{\Gamma((d+1) / 2)}{\Gamma(d / 2)} \frac{N_{C(d-2) / 2}}{n_{(d-2) / 2}} \mathscr{J}_{\frac{(d-1)}{2}}\left(\eta_{d}\right)
$$

Where $\mathrm{N}_{\mathrm{C}(d-2) / 2}$ is the effective density of states for the conduction band with $\mathrm{m}^{*}$ now being the density-ofstates effective mass. $\mathrm{n}_{(d-2) / 2}$ is the carrier concentration per unit volume(area or length).

\section{Bulk Semiconductors}

In bulk semiconductors all three Cartesian directions are much larger than the De Broglie wavelength. Therefore energy spectrum is analog-type in $x, y$ and $z$-direction as given by

$$
E_{k}=E_{c o}+\frac{\hbar^{2}}{2 m}\left(k_{x}^{2}+k_{y}^{2}+k_{z}^{2}\right)
$$

With the eigenfunction $\psi_{k}(\vec{r})$

$$
\psi_{k}(x, y, z)=\frac{1}{\sqrt{\Omega}} e^{j(\vec{k} \cdot \vec{r})}=\frac{1}{\sqrt{\Omega}} e^{j\left(k_{x} \cdot x+k_{y} \cdot y+k_{z} \cdot z\right)}
$$

Here $\mathrm{k}_{\mathrm{x}, \mathrm{y}, \mathrm{z}}$ are the wave-vector components with momentum $\vec{p}=\hbar \vec{k} . E_{c o}$ is the unaltered conduction band edge, $m^{*}$ is the carrier effective mass assumed isotropic for all three dimensions, $\Omega=\mathrm{L}_{\mathrm{x}} \mathrm{L}_{\mathrm{y}} \mathrm{L}_{\mathrm{z}}$ is the volume of the samples with $L_{x, y, z}$ the length in each of the three Cartesian directions.

Figure 2 indicates the ultimate velocity as a function of temperature for three values of concentration for 3D bulk silicon. Also shown is the graph for nondegenerate approximation. The velocity for low carrier concentration follows $T^{1 / 2}$ behaviour independent of carrier concentration. However for high concentration (degenerate carriers) the velocity depends strongly on concentration and becomes independent of the temperature. The ultimate saturation velocity is thus the thermal velocity appropriate for 3D carrier motion [6]:

$$
v_{i 3 N D}=v_{t h 3}=\frac{2}{\sqrt{\pi}} v_{t h}=\sqrt{\frac{8 k_{B} T}{\pi m^{*}}} \quad \text { (Nondegenerate) }
$$




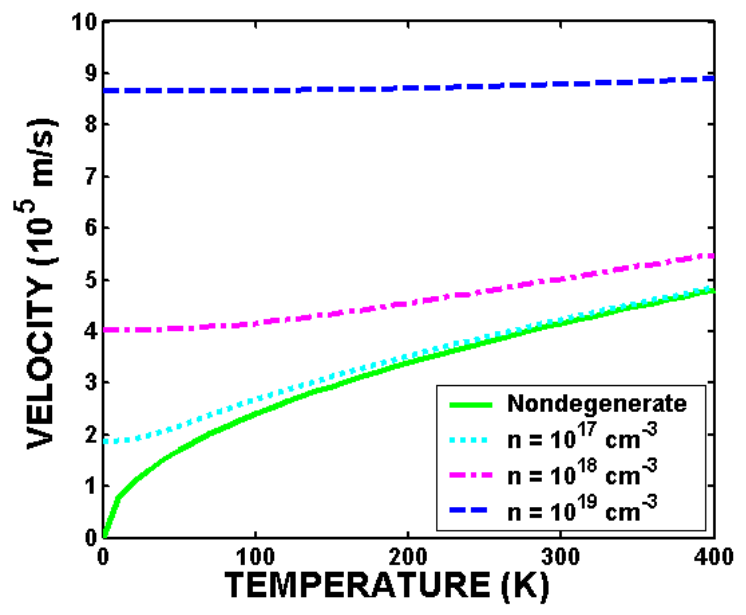

Figure 2: Velocity versus temperature for silicon for various concentration values.

Figure 3 shows the graph of ultimate intrinsic velocity as a function of carrier concentration for three temperatures $(\mathrm{T}=4.2 \mathrm{~K}, 77 \mathrm{~K}$, and $300 \mathrm{~K})$. As expected, at low temperature, carriers follow the degenerate statistics and hence their velocity is limited by appropriate average of the Fermi velocity that is a function of carrier concentration. When degenerate expression for the Fermi energy as a function of carrier concentration is utilized, the ultimate saturation velocity for bulk semiconductor (3D) is given by [7]

$$
v_{i 3 D}=\frac{3 \hbar}{4 m^{*}}\left[3 \pi^{2} n\right]^{\frac{1}{3}} \text { (Degenerate) }
$$

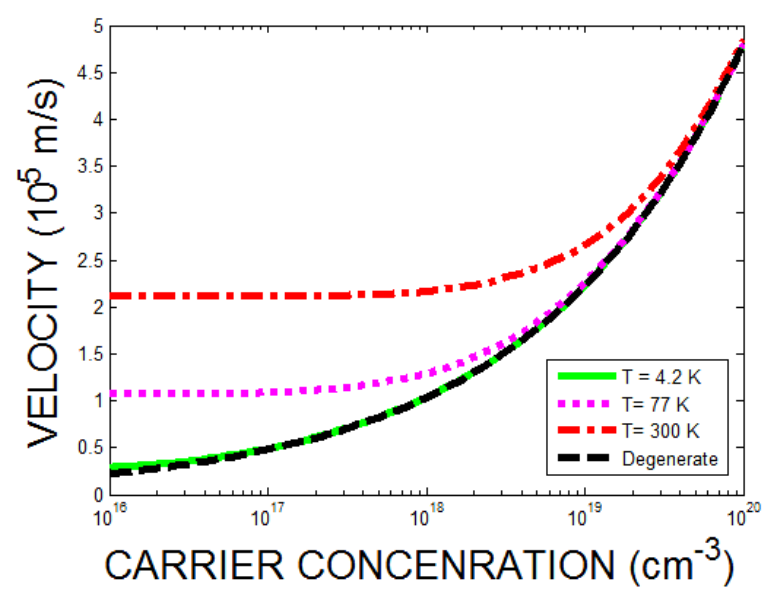

Figure 3: Velocity versus doping concentration for $\mathrm{T}=4.2 \mathrm{~K}$ (liquid helium), $\mathrm{T}=77 \mathrm{~K}$ (liquid nitrogen) and $\mathrm{T}=300 \mathrm{~K}$ (room temperature). The $4.2 \mathrm{~K}$ curve is closer to the degenerate limit. 


\section{Two Dimensional (2D) Semiconductors}

In 2D semiconductors only two Cartesian directions are much larger than the De - Broglie wavelength $\lambda_{\mathrm{D}}$. Therefore energy spectrum is analog-type in $\mathrm{x}, \mathrm{y}$-direction as given by.

$$
\begin{aligned}
& E=E_{c o}+\frac{\hbar^{2}}{2 m_{e}^{*}}\left(k_{x}^{2}+k_{y}^{2}\right)+n^{2} \in_{o z e} \\
& \in_{o z e(h)}=\frac{\pi^{2} \hbar^{2}}{2 m_{e(h)}^{*} L_{z}^{2}}
\end{aligned}
$$

The $\in_{\text {oze }(h)}$ is quantum limits in $z$ direction for both electron and holes in which the length $\mathrm{L}_{Z} \leq \lambda_{\mathrm{D}} \approx 10 \mathrm{~nm}$. The eigen-function $\psi_{k}(\vec{r})$ is given by

$$
\psi_{k}(x, y, z)=\frac{1}{\sqrt{L_{x} L_{y}}} e^{j\left(k_{x} \cdot x+k_{y} \cdot y\right)} \sqrt{\frac{2}{L_{z}}} \operatorname{Sin}\left(\frac{n \pi}{L_{z}} z\right)
$$

This wave-function describes the propagating waves in $\mathrm{x}$ and $\mathrm{y}$ directions. For quasi-2D nanostructures $\mathrm{d}=$ 2 , the ultimate average velocity per electron is now $v_{i 2}$ and is a function of temperature and doping concentration

$$
\begin{aligned}
& v_{i}=\sqrt{\frac{8 k_{B} T}{\pi m^{*}}} \times \frac{N_{C 2}}{n_{2}} \mathscr{J}_{0}\left(\eta_{F 2}\right) \\
& N_{C 2}=\frac{m * k_{B} T}{\pi \hbar^{2}}
\end{aligned}
$$

Figure 4 indicates the ultimate velocity as a function of temperature. Also shown is the graph for nondegenerate approximation. The velocity for low carrier concentration follows $T^{1 / 2}$ behaviour independent of carrier concentration. However for high concentration (degenerate carriers) the velocity depends strongly on concentration and becomes independent of the temperature. The ultimate saturation velocity is thus the thermal velocity appropriate for $2 \mathrm{D}$ carrier motions same as $3 \mathrm{D}$.

$$
v_{i 2 N D}=v_{t h 2}=v_{t h} \frac{\Gamma\left(\frac{3}{2}\right)}{\Gamma(1)}=\sqrt{\frac{\pi k_{B} T}{2 m^{*}}} \quad \text { (Non-degenerate) }
$$




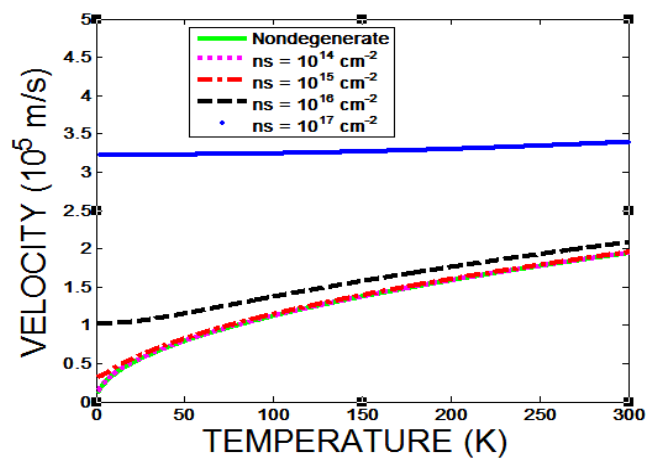

Figure 4: Velocity versus temperature for silicon for various concentration values.

Figure 5 shows the graph of ultimate intrinsic velocity as a function of carrier concentration for three temperatures ( $\mathrm{T}=4.2 \mathrm{~K}, 77 \mathrm{~K}$, and $300 \mathrm{~K})$. As expected, similar to bulk semiconductor (3D) at low temperature, carriers follow the degenerate statistics and hence their velocity is limited by appropriate average of the Fermi velocity that is a function of carrier concentration. When degenerate expression for the Fermi energy as a function of carrier concentration is utilized, the ultimate saturation velocity is given by

$$
v_{i 2 D}=\frac{2 \hbar}{3 m} \sqrt{2 \pi n_{2}} \quad \text { (Degenerate) }
$$

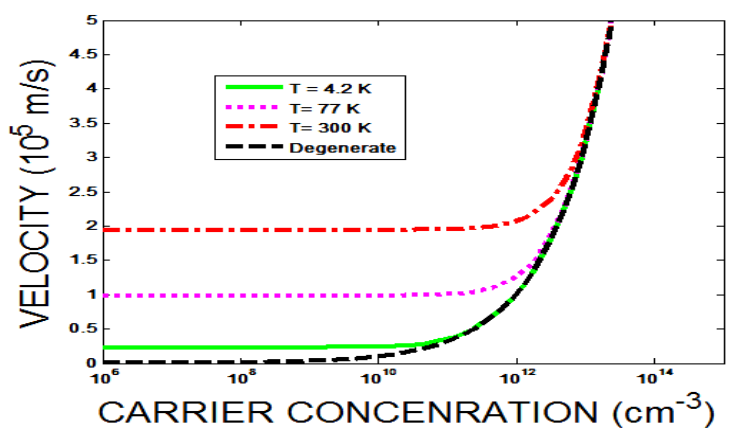

Figure 5: Velocity versus doping concentration for $\mathrm{T}=4.2 \mathrm{~K}$ (liquid helium), $\mathrm{T}=77 \mathrm{~K}$ (liquid nitrogen) and $\mathrm{T}=300 \mathrm{~K}$ (room temperature). The $4.2 \mathrm{~K}$ curve is closer to the degenerate limit.

\section{One Dimensional Semiconductor}

In one dimensional semiconductor only one Cartesian direction are much larger than the De -Broglie wavelength. Therefore energy spectrum is analog-type in $\mathrm{y}, \mathrm{z}$-direction as given by.

$$
E=E_{c o}+\frac{\hbar^{2}}{2 m_{e}^{*}}\left(k_{x}^{2}\right)+n^{2} \in_{\text {oze }}+m^{2} \in_{\text {oye }} m, n=1,2,3, \ldots \ldots .
$$


Here $\epsilon_{\text {oze( } \mathrm{h})}=\pi^{2} \hbar^{2} / 2 \mathrm{~m}_{\mathrm{e}(\mathrm{h})}^{*} \mathrm{~L}_{\mathrm{z}}^{2}$ and $\epsilon_{\text {oye( } \mathrm{(h})}=\pi^{2} \hbar^{2} / 2 \mathrm{~m}_{\mathrm{e}(\mathrm{h})}^{*} \mathrm{~L}_{\mathrm{y}}^{2}$ are digital type in $\mathrm{x}$ direction. With the eigenfunction $\psi_{k}(\vec{r})$ given by

$$
\psi_{k}(x, y, z)=\frac{1}{\sqrt{L_{x}}} e^{j\left(k_{x} \cdot x\right)} \sqrt{\frac{2}{L_{z}}} \operatorname{Sin}\left(\frac{n \pi}{L_{z}} z\right) \sqrt{\frac{2}{L_{y}}} \operatorname{Sin}\left(\frac{m \pi}{L_{y}} y\right)
$$

This wave function describes the propagating waves in one direction. For quasi-one-dimensional $d=1$, the ultimate average velocity per electron is now $v_{i 1}$ and is a function of temperature and doping concentration too.

$$
v_{i}=\sqrt{\frac{2 k_{B} T}{\pi m^{*}}} \times \frac{N_{C 1}}{n_{1}} \mathscr{J}_{0}\left(\eta_{F 1}\right)
$$

With

$$
N_{c 1}=\left(\frac{2 m * k_{B} T}{\pi \hbar^{2}}\right)^{\frac{1}{2}}
$$

Where $N_{C 1}$ is the effective density of states for the conduction band with $\mathrm{m}^{*}$ now being the density-ofstates effective mass. $n_{1}$ is the carrier concentration per unit length. Figure 6 indicates the ultimate velocity as a function of temperature. Also shown is the graph for nondegenerate approximation. Same as 3D and 2D devices the velocity for low carrier concentration follows $T^{1 / 2}$ behaviour independent of carrier concentration. However for high concentration (degenerate carriers) the velocity depends strongly on concentration and becomes independent of the temperature. The ultimate saturation velocity is thus the thermal velocity appropriate for 1D carrier motion:

$$
v_{i 1 N D}=v_{t h 1}=\frac{1}{\sqrt{\pi}} v_{t h}=\sqrt{\frac{2 k_{B} T}{\pi m^{*}}} \text { (Nondegenerate) }
$$

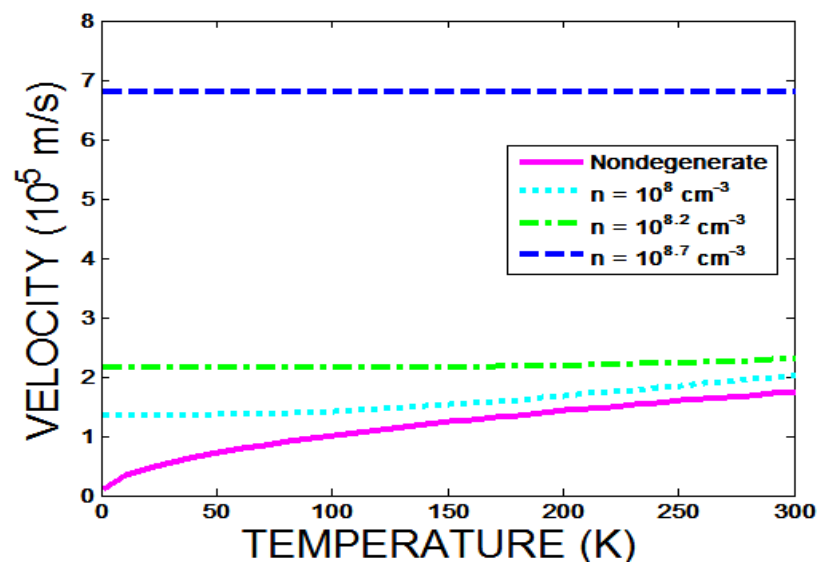

Figure 6: Velocity versus temperature for nanowire for various concentration values. 
Figure 7 shows the graph of ultimate intrinsic velocity as a function of carrier concentration for three temperatures $(\mathrm{T}=4.2 \mathrm{~K}, 77 \mathrm{~K}$, and $300 \mathrm{~K})$. As expected, similar to $3 \mathrm{D}$ and $2 \mathrm{D}$ devices at low temperature, carriers follow the degenerate statistics and hence their velocity is limited by appropriate average of the Fermi velocity that is a function of carrier concentration. When degenerate expression for the Fermi energy as a function of carrier concentration is utilized, the ultimate saturation velocity is given by

$$
v_{i 1 D}=\frac{\hbar}{4 m^{*}}\left(n_{1} \pi\right) \text { (Degenerate) }
$$

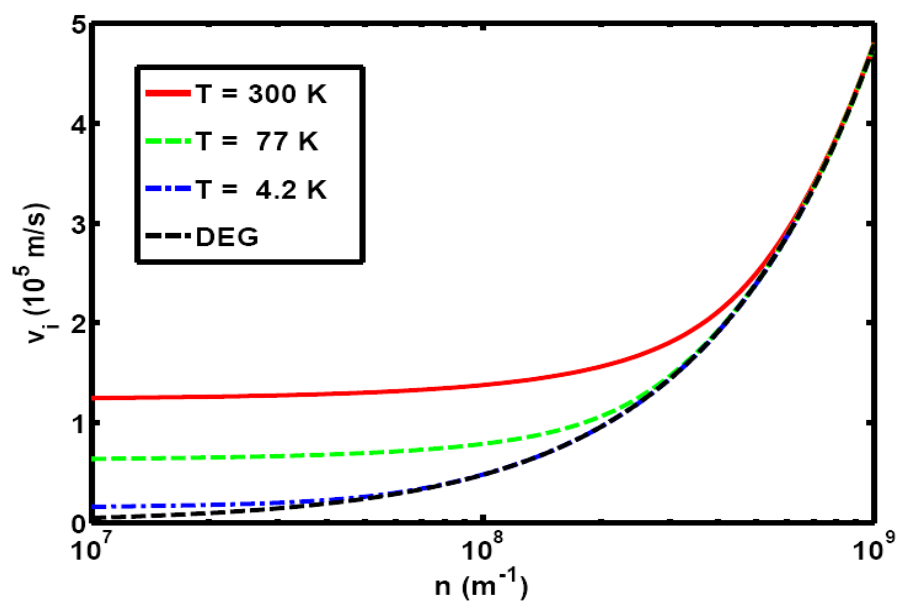

Figure 7: Velocity versus doping concentration for $\mathrm{T}=4.2 \mathrm{~K}$ (liquid helium), $\mathrm{T}=77 \mathrm{~K}$ (liquid nitrogen) and $\mathrm{T}=300 \mathrm{~K}$ (room temperature). The $4.2 \mathrm{~K}$ curve is closer to the degenerate limit.

The ultimate velocity in all dimensions may become lower when quantum emission is considered. The inclusion of the quantum or optical phonon or any other similar emission will change the temperature dependence of the saturation velocity.

\section{Conclusion}

Using the distribution function that takes into account the asymmetrical distribution of drifting electrons in an electric field is presented. This distribution function transforms the random motion of electrons into a streamlined one that gives the ultimate saturation velocity that is a function of temperature in nondegenerate regime and a function of carrier concentration in the degenerate regime. The ultimate drift velocity is found to be appropriate thermal velocity for a given dimensionality for no degenerately doped samples. However, the ultimate drift velocity is the appropriate average of the Fermi velocity for degenerately doped samples.

\section{Acknowledgement}

The authors would like to thank the Ministry of Science, Technology and Industry (MOSTI) and RMC for research grant. VKA would like to acknowledge the excellent hospitality of the Universiti Teknologi Malaysia (UTM). 


\section{References}

[1] V. K. Arora, Japanese Journal of Applied Physics, 24, 537(1985).

[2] Vijay K. Arora, Applied Physics Letters, 80, 3763(2002).

[3] V. K. Arora and Michel L.P.Tan, Ismail Saad Razali Ismail Applied Physics Letters (2007)

[4] Vijay K. Arora, proceedings of IEEE international conference on Microelectronics,Belgrade,Serbia and Montenegro,14-17 May 2006(IEEE,New York,2006)pp.17-24

[5] A.M.T.Fairus and V.K.Arora Microelectron.J.32, 679(2001)

[6] Nanoscale Transistors Device Physics, Modeling and Simulation. Jing Guo, Mark S. Lundestorm Spriger (2006)

[7] Vijay K. Arora.Jpn.J, Applied Physics Letters, part 1 24,537(1985) 